\title{
Spectral library searching to identify cross-linked peptides.
}

Devin K Schweppe ${ }^{1}$, Juan D Chavez ${ }^{1}$, Arti T Navare ${ }^{1}$, Xia Wu ${ }^{1}$, Bianca Ruiz ${ }^{1}$, Jimmy K Eng ${ }^{2}$, Henry Lam $^{3}$, James E Bruce ${ }^{*}$

${ }^{1}$ Department of Genome Sciences

University of Washington School of Medicine

Seattle, WA 98195, USA.

${ }^{2}$ University of Washington Proteomics Resource

University of Washington School of Medicine

Seattle, WA 98195, USA.

${ }^{3}$ Department of Chemical and Biomolcular Engineering

Hong Kong University of Science and Technology

Clear Water Bay, Kowloon, Hong Kong

\section{Supporting Information Table of Contents}

\section{Supporting Tables}

Table S-1. Spectra-spectra-match information for the 157 relationships SpectraST identified from the library-query that were not identified by ReACT (see Figure S-3 for reciprocal plots).

\section{Supporting Figures}

Figure S-1. Characterization of FDR estimation methods.

Figure S-2. Detailed analysis based on individual cases of false negatives/false positives of those identifications that SpectraST searching was unable to recapitulate and description of potential causes.

Figure S-3. Reciprocal plots for the 157 relationships SpectraST identified from the library-query that were not identified by ReACT (see Table S-1 for relationship information).

Figure S-4. NCE optimization and example spectra for QE+ query searches.

Figure S-5. Example spectra for in vivo Klebsiella relationships queried against cryo-crosslinking library.

Figure S-6. SpectraST identifications for non-cleavable cross-linked protein analysis based on public data.

Figure S-7. SpectraST search instructions.

Figure S-8. Comparison of decoy-free binning versus peak-to-peak selection and PeptideProphet distributions. 


\section{Supporting Information Legends}

Supporting Tables

Table S-1. Spectra-spectra match information for the 157 relationships SpectraST identified from the library-query that were not identified by ReACT (see Figure S-3 for reciprocal plots).

\section{Supporting Figures}

Figure S-1. Characterization of FDR estimation methods. (A) Total correct SSMs versus FDR for the dot-product filter, precursor-swap, and decoy-free filtering methods. (B) Comparison of total nonredundant relationships identified by either decoy-free ( $5 \%$ FDR) or dot-product filtering $(>0.5)$.

Figure S-2. Detailed analysis based on individual cases of false negatives/false positives of those identifications that SpectraST searching was unable to recapitulate and description of potential causes. (A) Case A, one-peptide correct: one peptide was different between SpectraST and ReACT identifications. (B) Case B, Poor SSM matching: the SpectraST match fell below dot-product $=0.2$ and was dropped during PeptideProphet analysis. (C) Case C, lysine-site shift: the SpectraST identified site of cross-linking was different than the ReACT identified site. (D) Case D, false positives: SSMs that differed from ReACT, but the SpectraST match passed PeptideProphet filtering.

Figure S-3. Reciprocal plots for the 157 relationships SpectraST identified from the library-query that were not identified by ReACT (see Table S-1 for relationship information).

Figure S-4. NCE optimization and example spectra for QE+ query searches. (A) Optimization of NCE from HCD NCE $=5-80$ using cross-linked bradykinin. (B) Three relationships with their corresponding reciprocal plots and precursor $\mathrm{m} / \mathrm{z}$ 's are shown.

Figure S-5. Example spectra for in vivo Klebsiella relationships queried against cryo-crosslinking library. Three relationships with their corresponding reciprocal plots and precursor $\mathrm{m} / \mathrm{z}$ 's are shown.

Figure S-6. SpectraST identifications for non-cleavable cross-linked protein analysis based on public data. (A) Workflow for analysis of non-cleavable cross-linkers with SpectraST searching. Data used for the analysis were taken from publicly available data (ProteomeXchange: PXD001454). (B) PeptideProphet distributions for correct and incorrect SSMs.

Figure S-7. SpectraST search instructions.

Figure S-8. Comparison of decoy-free binning versus peak-to-peak selection and PeptideProphet distributions. (A) Total number of correct hits at a given FDR for four different SpectraST bin settings. No binning was the most conservative binning parameter. (B) PeptideProphet distributions for correct and incorrect SSMs for SLAb queries. 


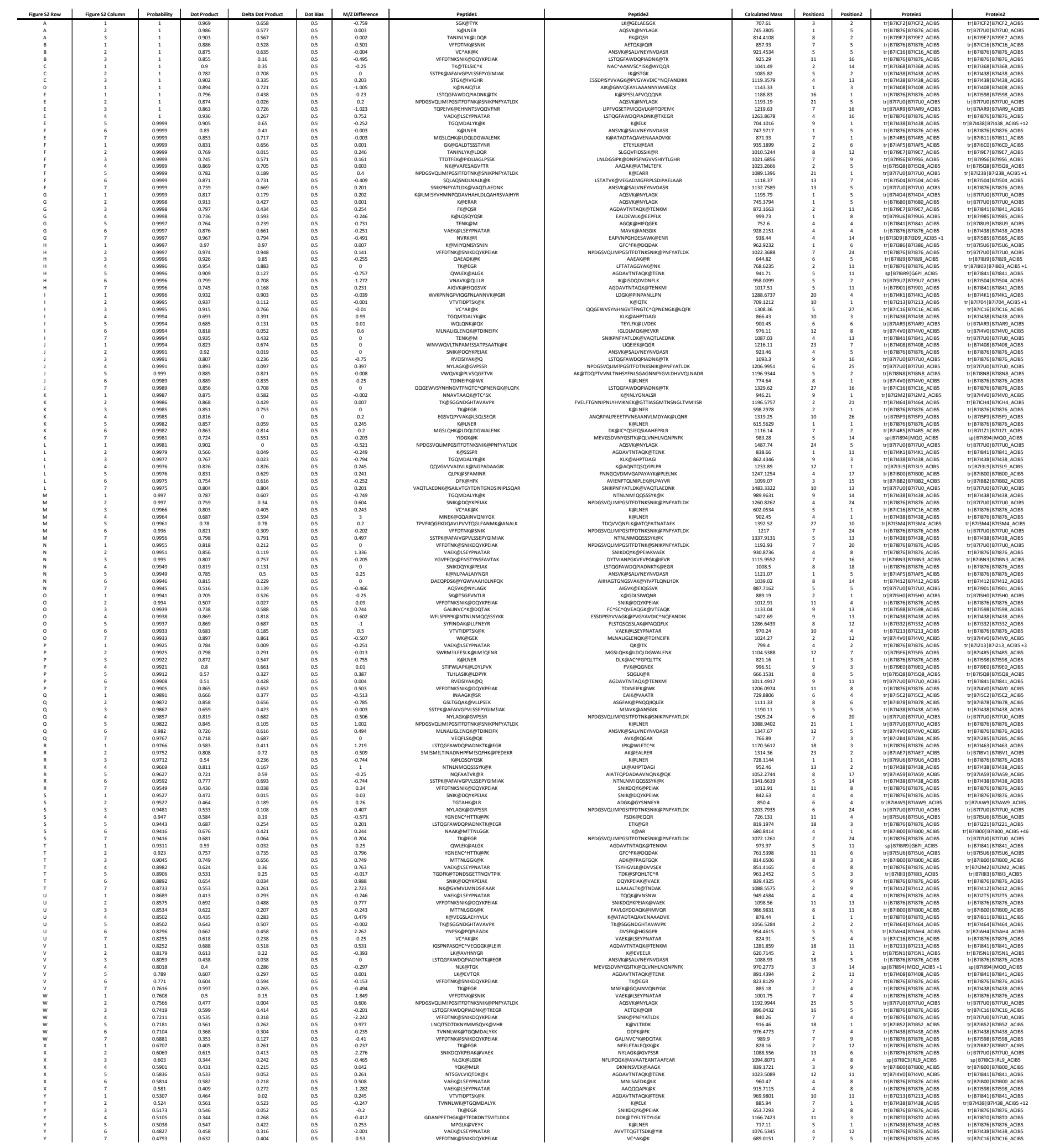


Figure S1

a

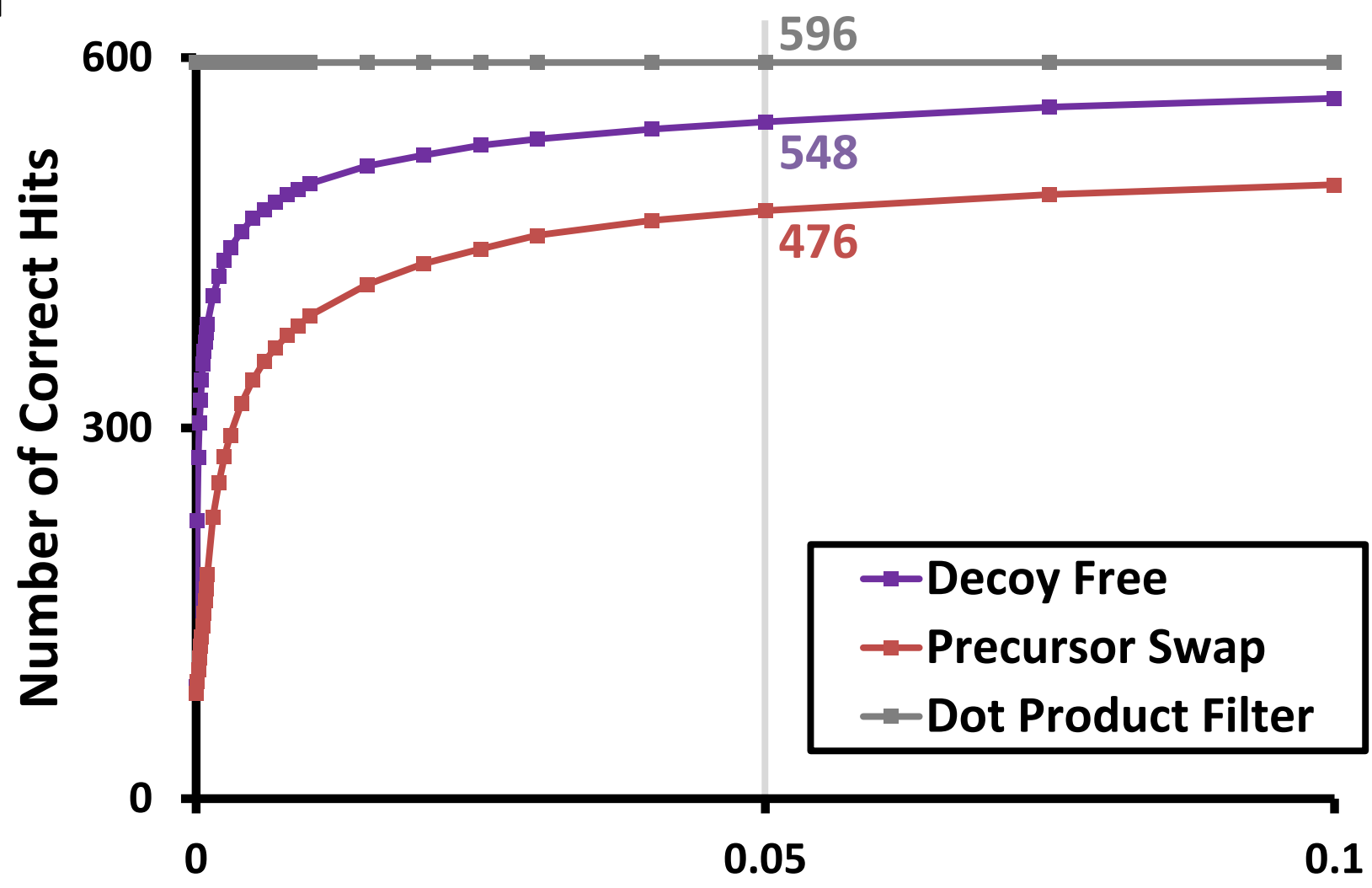

False Discovery Rate

b

SpectraST:

$n=445$

(dp $>0.5$ )
Intersection of non-

redundant Relationships

$$
n=403
$$

SpectraST:

$n=419$

(DF-FDR < 5\%) 


\section{Figure S2}

\section{8 relationships not ID'd by SpecraST (FDR < 5\%)}

10 non-redundant ReACT relationships recovered at 10\% FDR (FN)

18 non-redundant ReACT relationships not matched by SpectraST

\begin{tabular}{|c|c|c|c|}
\hline Case & Description (FP or FN) & \# Mismatched spectra & Potential Causes \\
\hline A & One peptide difference (FP) & $\mathbf{7 / 1 8}$ & $\begin{array}{c}\text { MS2 spectral matching biased } \\
\text { for one peptide }\end{array}$ \\
\hline B & SpectraST dot product <0.2 (FN) & $\mathbf{7 / 1 8}$ & $\begin{array}{c}\text { Poor matching spectra, e.g. } \\
\text { precursor charge state } \\
\text { different in library v. query }\end{array}$ \\
\hline C & Lysine-site shift within peptide & $\mathbf{1 / 1 8}$ & Ambiguous at MS level \\
\hline D & $\begin{array}{c}\text { Same scan match passed } \\
\text { Prophet with wrong ID (FP) }\end{array}$ & $\mathbf{3 / 1 8}$ & Incorrect ID \\
\hline
\end{tabular}

FN - False Negative

False negative rate at least 20/419 (5\%)

A ${ }^{1 \mathbf{e + 8 4}} \triangle$ Peak differences

Library

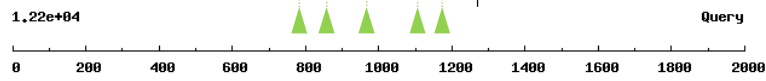

C SpectraST ID:

C VAEKLSEYPNATAR_LSTQGFAWDQPIADNKTKEGR ReACT ID:

VAEKLSEYPNATAR_LSTQGEAWDQPIADNKTKEGR

\section{B \\ False}

FP - False Positive

alse positive rate at least 10/419 (2\%)

ReACT identification:

TKEGR_DQYKPEIAKVAEK

814.41 (ReACT MS ${ }^{2}$ )

$z=4$

\subsection{3 (Library MS²)}

$\mathbf{z}=5$

Library identification:

TKEGR_DQYKPEIAKVAEK
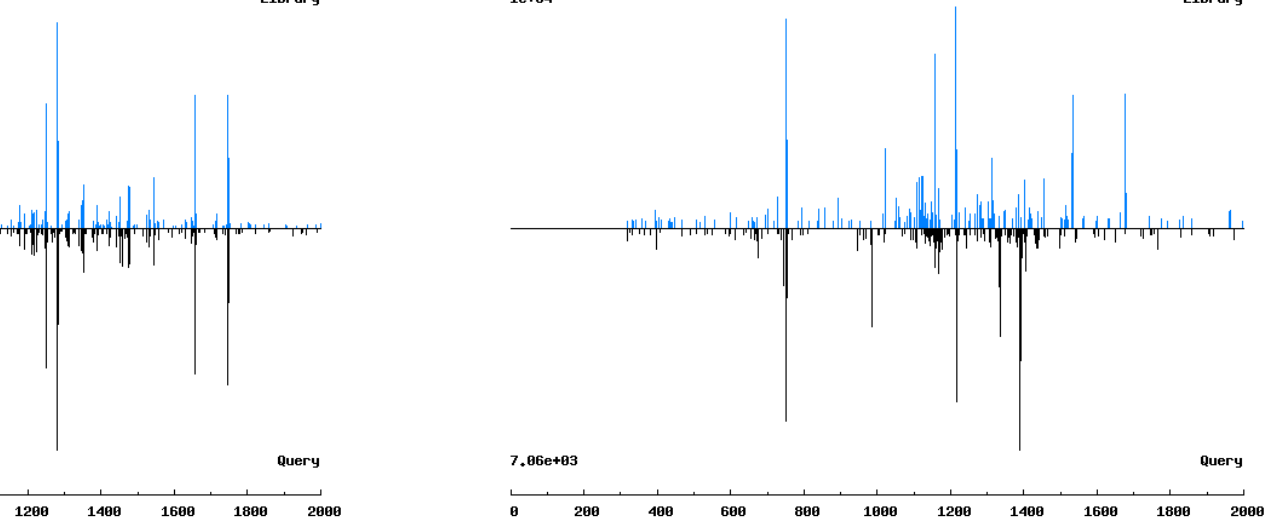
Figure S3
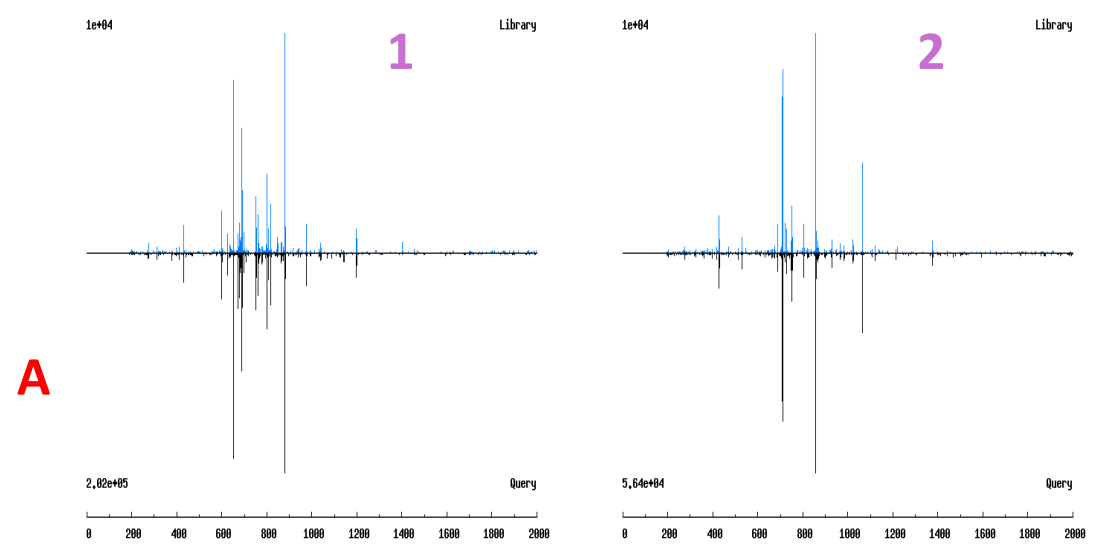

1e+84

Library

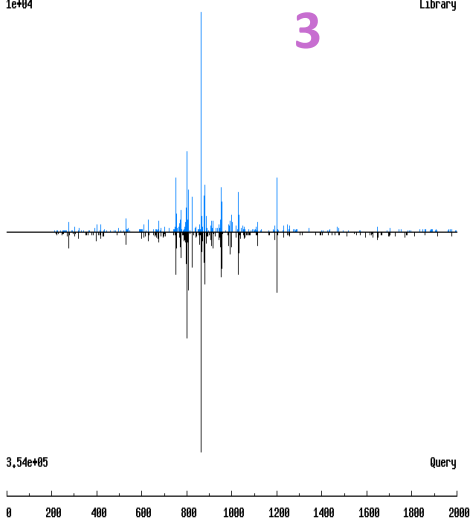

1et+84

Library

1eet84

Library

1 et+84

Library

B
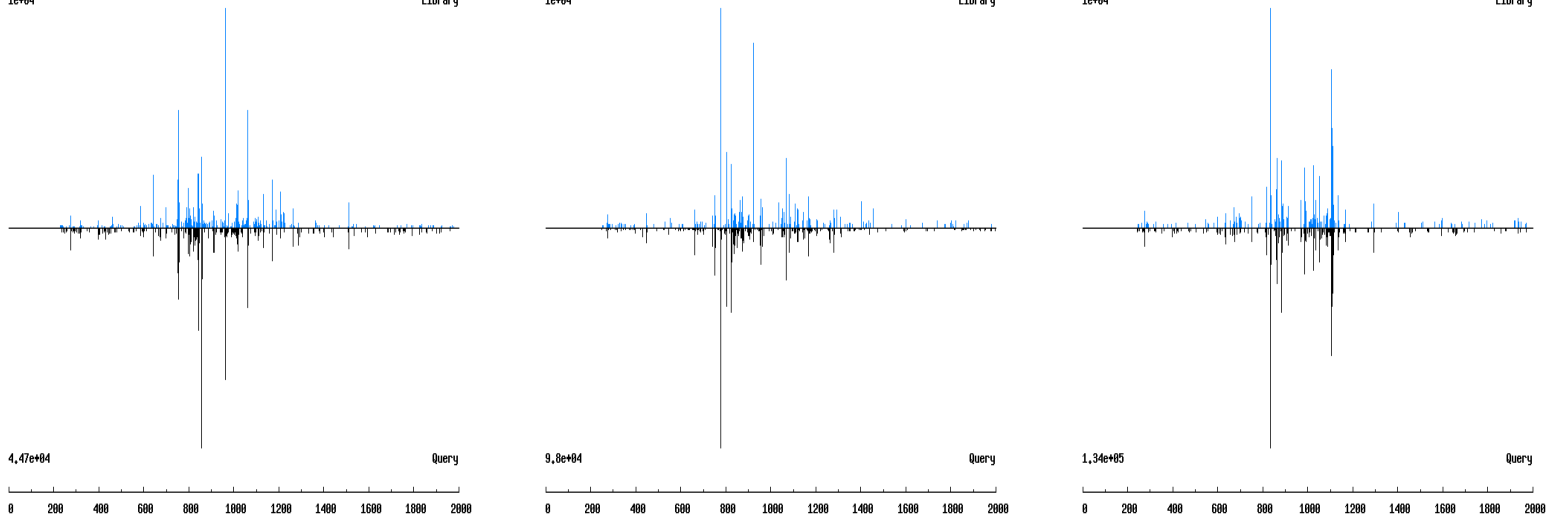

1e+84

Library

1et+84

Library

1et+84

Library

C

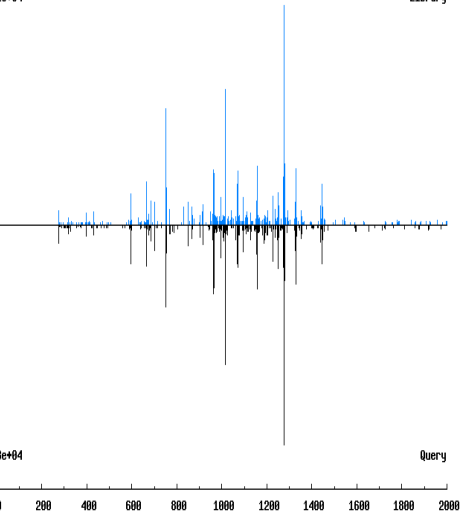

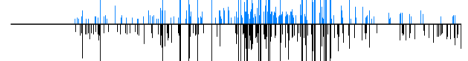

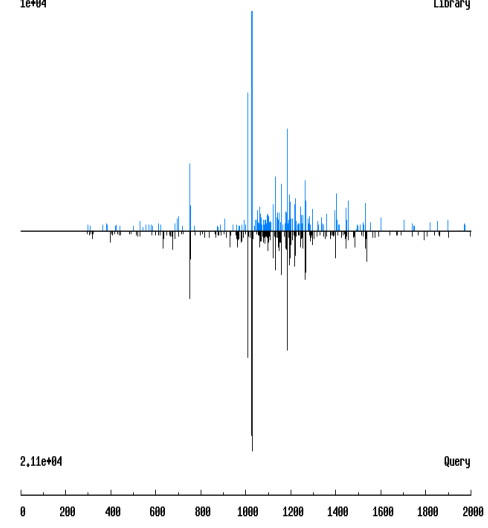

1 e+84

Library

D
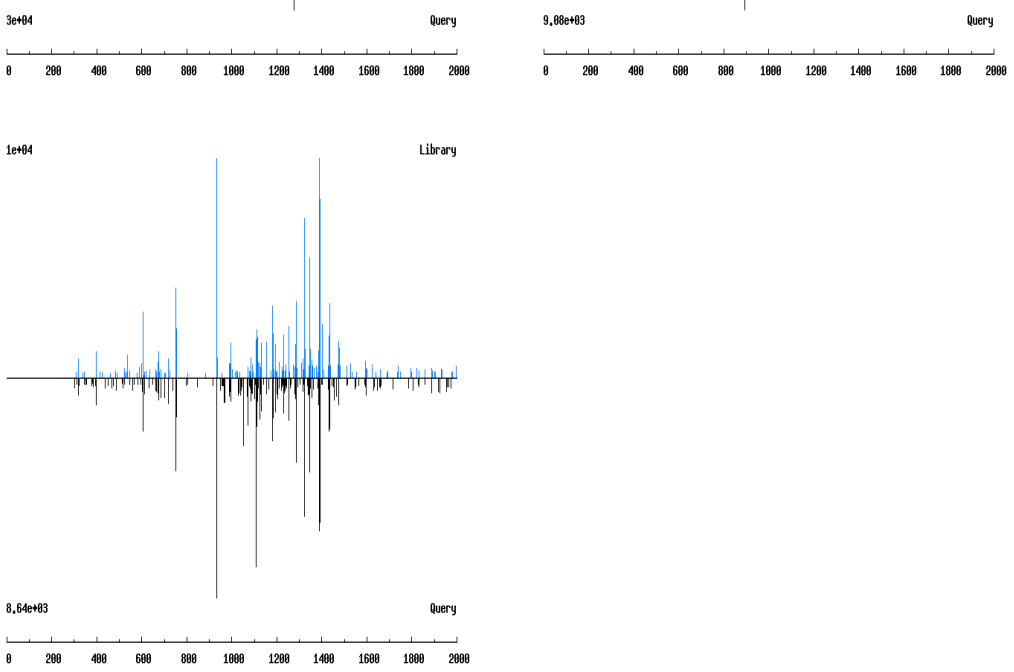


\section{Figure S4}

a Avg. NCE @ max intensity

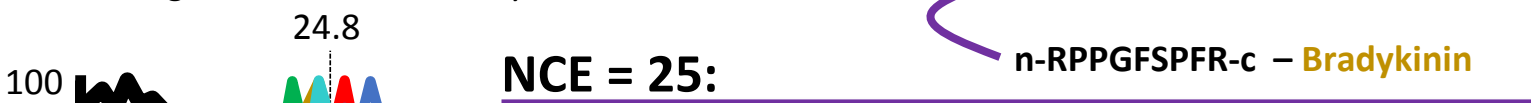

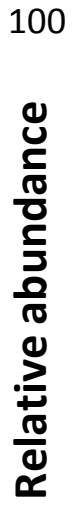
100 629.3061,z=2, released ( $\max$ int. @ NCE = 24)

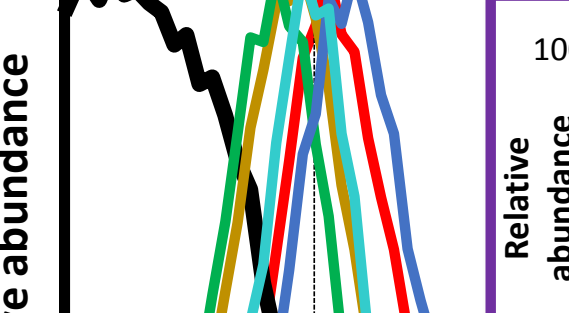

752.4152, z=1, reporter (max int. @ NCE =26)

817.4059, z=4, precursor

$\frac{\pi}{0}$

150

Thompsons

2000

b

P1: $\quad$ SRPC!K*IVDMSTSK

P2: $\quad$ K*LEDLSPSTHNMEVPVVK

$\mathrm{M} / \mathrm{Z}: \quad 1170.5800$

P1: VK*ALNAGK

P2: $\quad$ K*AGLNIVR

M/Z: $\quad 704.8800$
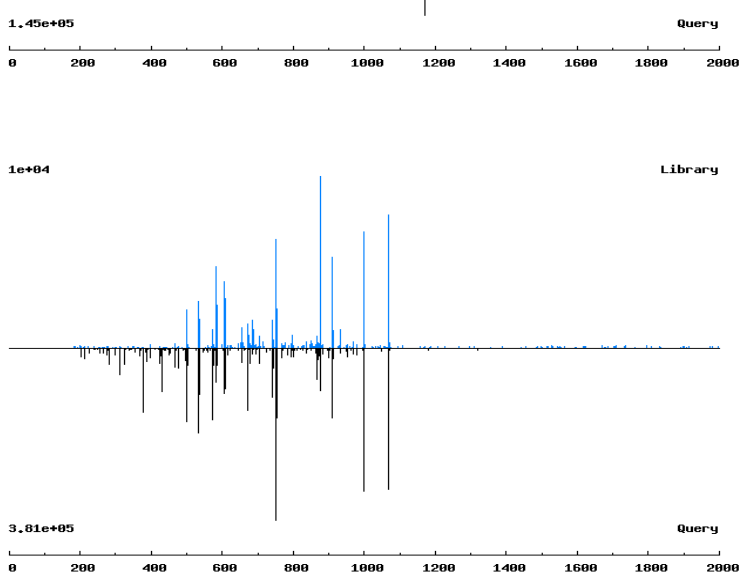

P1: SPIK*VVGLSSLPEIYEK

P2: M@EK*GQIAGR

M/Z: $\quad 1003.770$
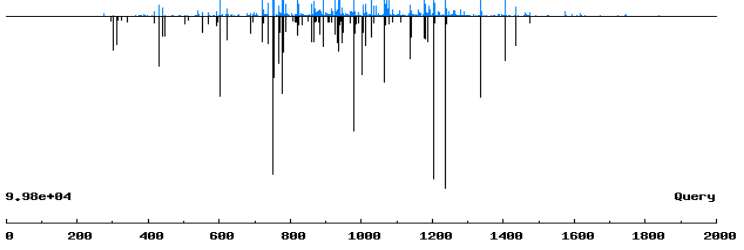


\section{Figure S5}

P1: $\quad K^{*}$ ATIELLNR

P2: AVQLGGVALGTTQVINSK*TPLQSYPLDIHHVQDHLK

M/Z: $\quad 1217.650$

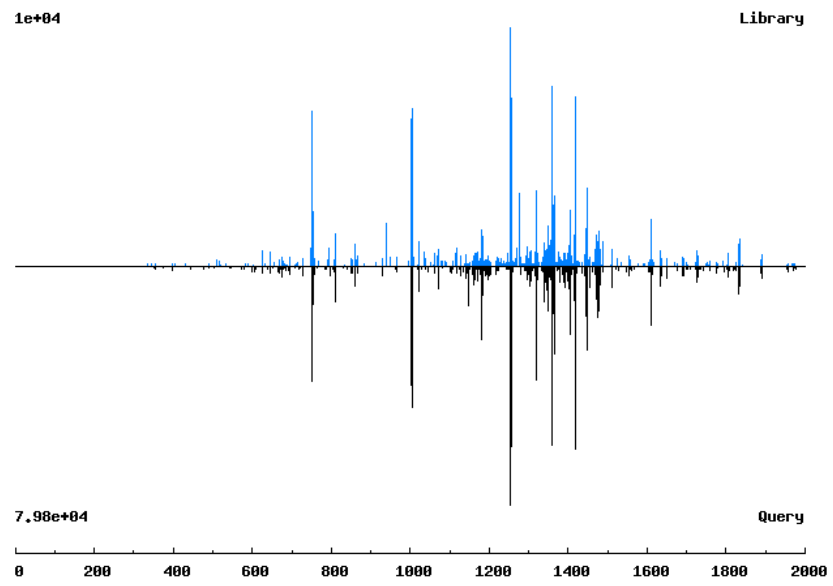

P1: HLAK*APAPAEAK

P2 LLAEHNLDPAAIK*GTGVGGR

$\mathrm{M} / \mathrm{Z}: \quad 1085.067$

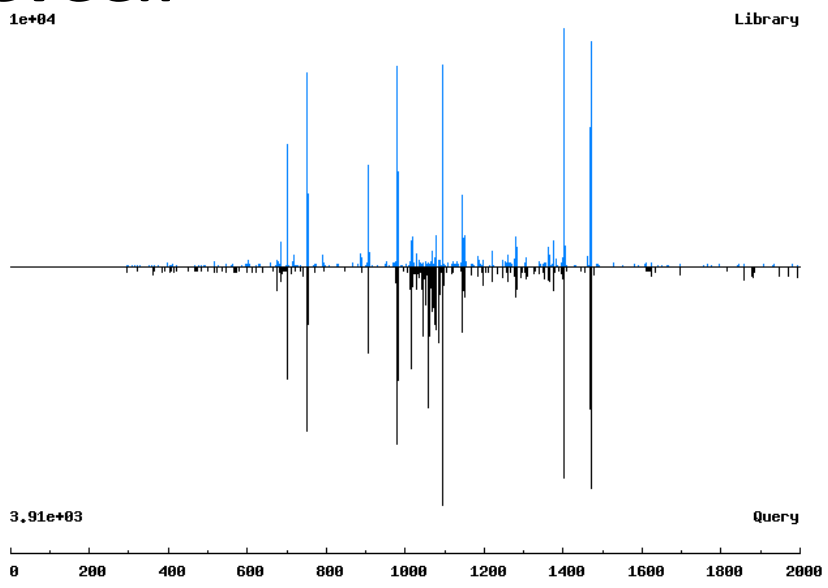

P1: K*AEQYLLENETTK teas

P2: $\quad K * A E Q Y L L E N E T T K$

M/Z: $\quad 1070.274$

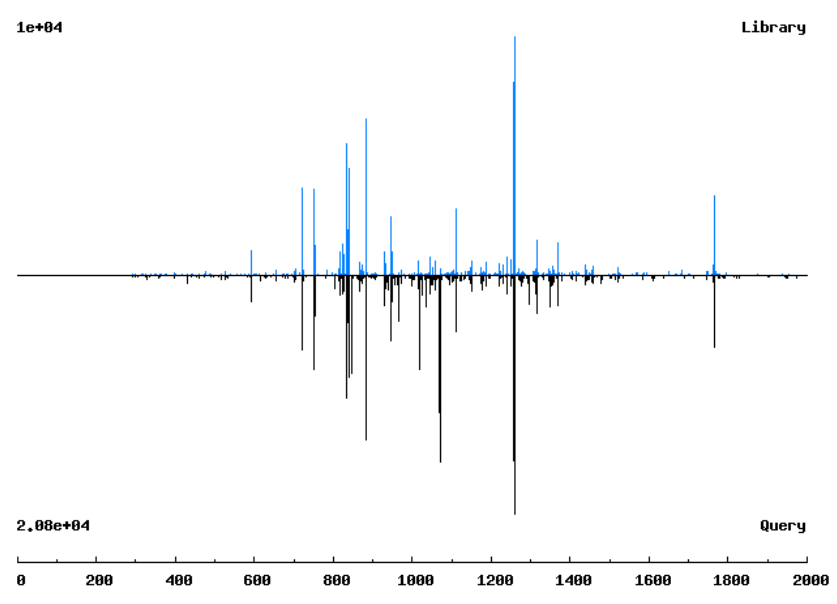




\section{Figure S6}

a
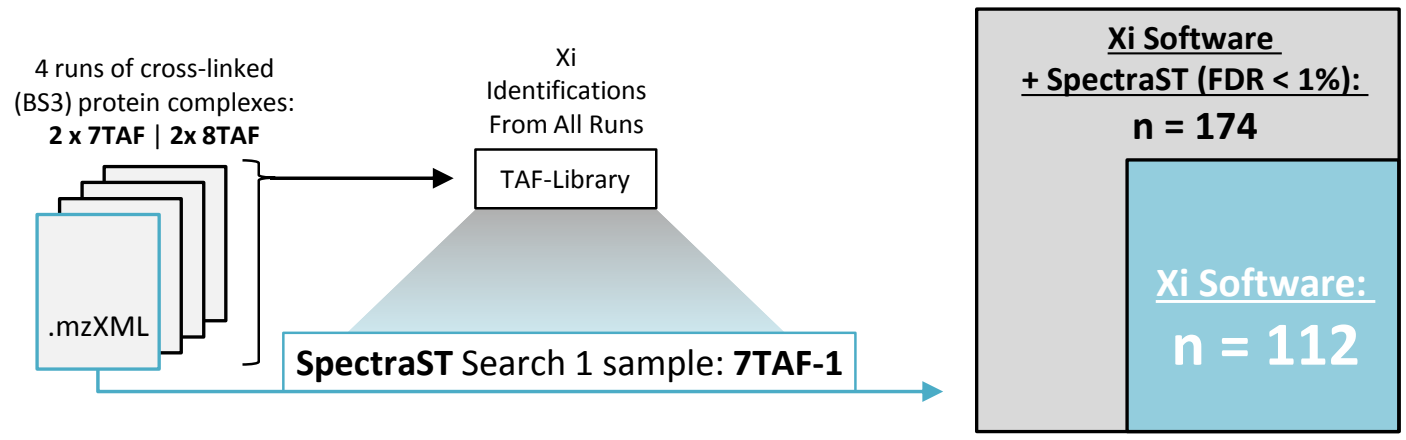

Original .raw filenames (ProteomeXchange: PXD001454):

1. Zou_Rappsilber_7TAF_2014_01.raw

2. Zou_Rappsilber_7TAF_2014_02.raw

$\mathrm{n}=$ non-redundant relationships

3. Zou_Rappsilber_8TAF_2014_01.raw

4. Zou_Rappsilber_8TAF_2014_02.raw

* MS and MS/MS spectra from high resolution Orbitrap scans.

b

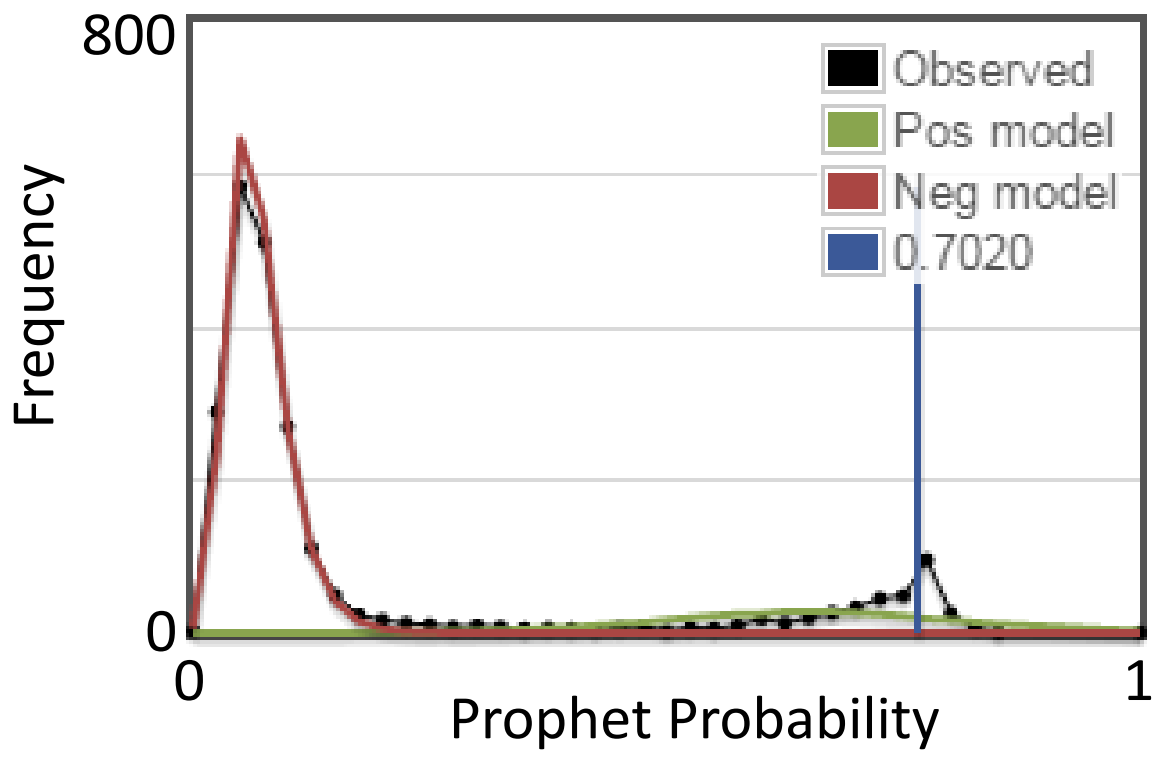




\section{SpectraST Search Instructions}

Within putty.exe command line:

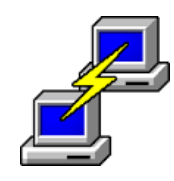

- $\quad$ Move to the directory containing your query mzXML files.

- $\quad$ Search using SpectraST:

spectrast -sz -NOB -sL/path/to/lib/file/test.spLib test1.mzXML

- $\quad$ Search using SpectraST with decoy-free method:

spectrast -sz -NOB -s_PVL -sL/path/to/lib/file/test.spLib test1.mzXML 


\section{Figure S8}

a

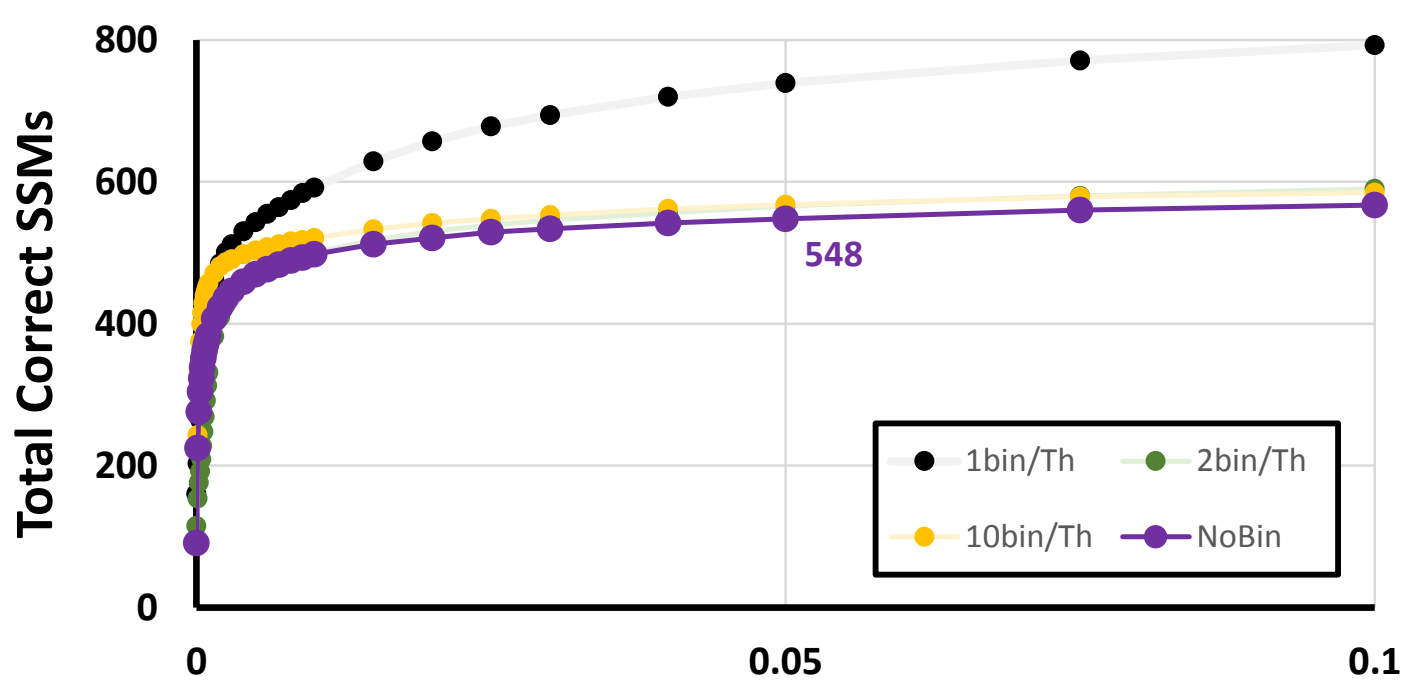

False Discovery Rate

b

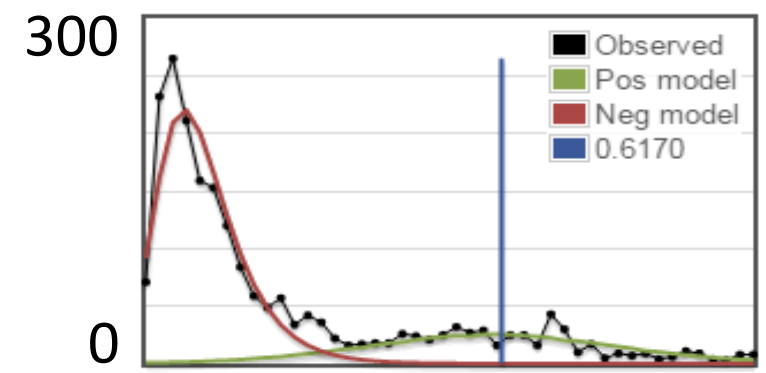

Library query

Library: SLAb

FDR Estimation: decoy-free

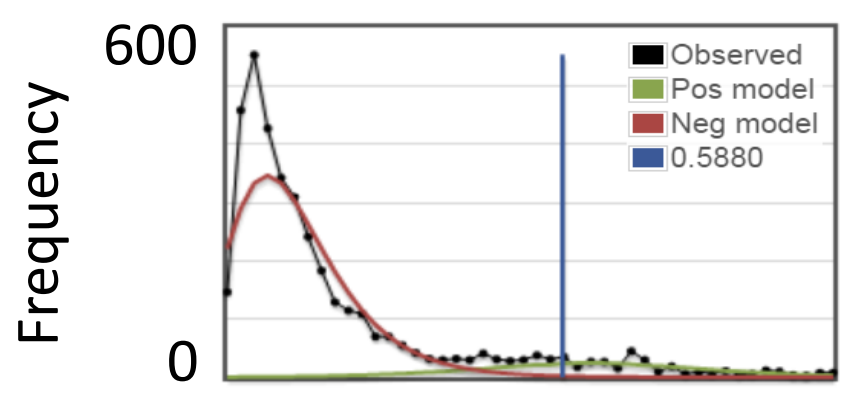

Library query

Library: SLAb

FDR Estimation: precursor swap

400

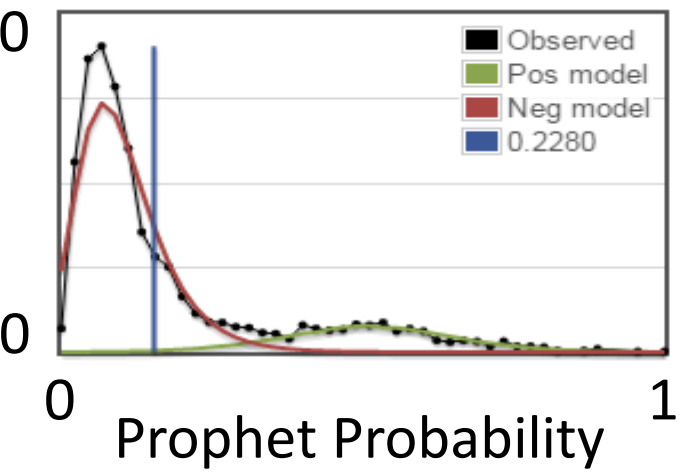

Replicate query

Library: SLAb

FDR Estimation: decoy-free 